\title{
Plaque Type of Porokeratosis of Mibelli Over Scalp with Squamous Cell Carcinoma of Tongue
}

\author{
Rochit Rajesh Singhal, Trusha Manoj Patel, Khushboo Hemant Modasia, Niral Ketan Sheth and Rita Vipul Vora* \\ Dept of Skin and VD, Shree Krishna Hospital, Karamsad, Anand, Gujarat (India)
}

\begin{abstract}
Porokeratosis of Mibelli is a benign, rare, genetic disorder of epidermal keratinization transmitted as an autosomal dominant trait. It is characterized by single or a few well formed annular or serpiginous lesions with central hyperpigmentation, mild atrophy and dryness with a characteristic peripheral, slightly raised, keratotic border. The histological hallmark of Porokeratosis is cornoid lamella which is a column of parakeratotic cells that invaginates the epidermis and corresponds to peripheral keratotic ridge. It is considered as premalignant condition. Here we report a case of 62 year old male with known case of squamous cell carcinoma of tongue presented with common plaque type porokeratosis of Mibelli over scalp without hairloss.
\end{abstract}

Keywords: Porokeratosis of Mibelli, Cornoid Lamella, Squamous Cell Carcinoma.

\section{Introduction}

Porokeratosis of Mibelli is a benign, rare, genetic disorder of epidermal keratinization transmitted as an autosomal dominant trait. Chronic sun exposure, Hepatitis B and $\mathrm{C}$ infection, HIV infection, organ transplantation and immunosuppression are important proposed factors in the clonal proliferation of keratinocytes. It is twice as common in males as in females. Onset is usually in childhood but the disease can occur at any age. It commonly develops on the extremities but has also been known to occur on the face, buccal mucosa, genitalia, palms and soles ${ }^{[1]}$. Here we report a case of 62 year old male with known case of squamous cell carcinoma of tongue presented with common plaque type porokeratosis of Mibelli over scalp without hairloss.

\section{Case Report}

A 62 year old male, farmer by occupation, with known case of squamous cell carcinoma of left side base of tongue for last 2 months was referred to the department of dermatology with complains of lesion over scalp which he noticed 15 days back. The lesion was associated with itching and pain. There was no history of trauma over that region. Family history of similar lesions was negative. Examination revealed a single well defined erythematous annular plaque of size $3 \times 3 \mathrm{~cm}$ with raised margins (beaded) of width $0.5 \mathrm{~cm}$ present over left parietal region of scalp with no hairloss[Figure 1]. Physical examination of other systems was unremarkable. Complete blood count, urinalysis, fasting blood sugar, liver and renal function were within normal limits and serologic tests for syphilis, HIV, hepatitis B and C were negative. Histopathological examination revealed keratin filled epidermal invagination with an angulated porokeratotic end [Figure 2(a)]. The epithelium adjacent and below the invagination was devoid of a granular cell layer and contained vacuolated keratinocytes [Figure 2(b)]. Adjacent epidermis was hyperkeratotic and acanthotic. Dermis showed a moderate lymphoplasmocytic infiltrate [Figure 2(a)]. No evidence of malignancy was noted in the studied section. Diagnosis of Porokeratosis of Mibelli was made on the basis of histopathological examination. Patient was operated for squamous cell carcinoma of base of tongue. For scalp lesion, he was given topical retinoid and was adviced for photoprotection and kept under regular follow up.

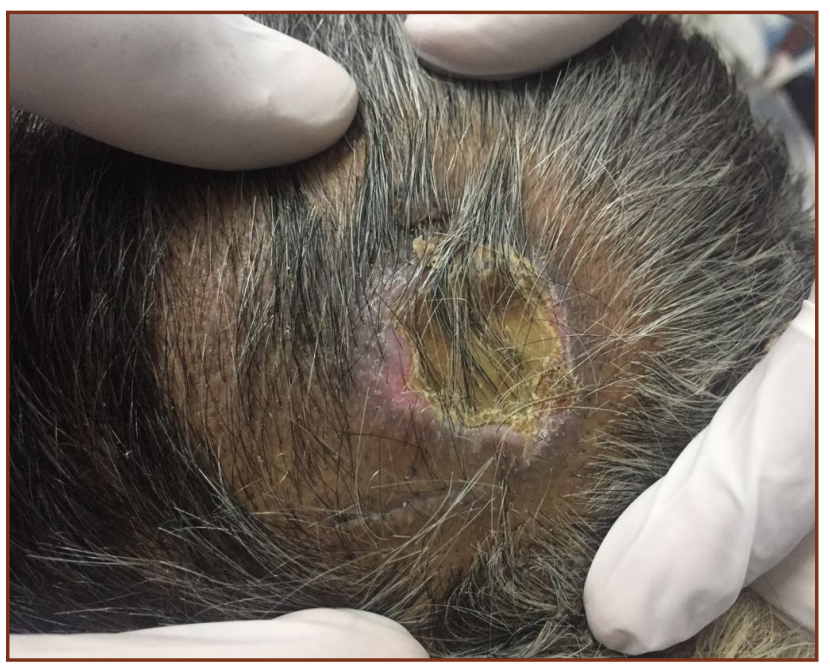

Fig. 1: single well defined erythematous annular plaque with central atrophy and raised margins (beaded) over left parietal region of scalp. 


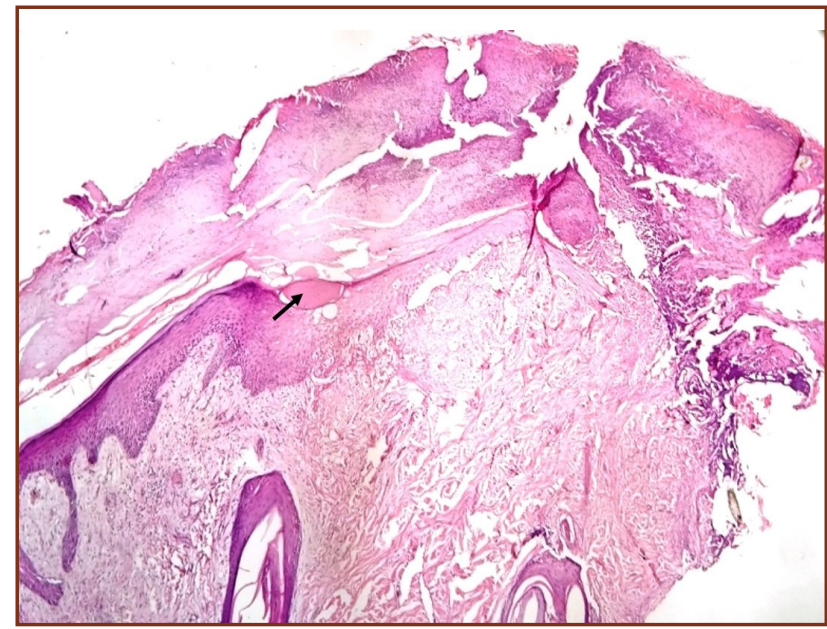

Fig. 2(a): Epidermis showing keratin filled invagination called cornoid lamella (highlighted with an arrow) (4X).

\section{Discussion}

The classic lesion of porokeratosis was first described by Mibelli in $1893^{[2]}$. He described plaque type as single or a few well formed annular or serpiginous lesions with central hyperpigmentation, mild atrophy and dryness with a characteristic peripheral, slightly raised, keratotic border. Other clinical variants reported are superficial disseminate, superficial actinic, linear, puntate, plaque and hypertrophic or warty types. Apart from these clinical variants, a number of atypical morphological forms such as facial porokeratosis, giant porokeratosis and reticulate porokeratosis have also been reported ${ }^{[3]}$. A patient may develop more than one type of porokeratosis simultaneously or consecutively. Giant porokeratosis is considered to be a morphological variant of porokeratosis of Mibelli with a diameter of up to $20 \mathrm{~cm}$ and surrounding wall of $1 \mathrm{~cm}$. It has high malignant potential. In our case, the lesion size was around $3 \mathrm{X} 3 \mathrm{~cm}$ with keratotic ridge of width $0.5 \mathrm{~cm}$.

The histological hallmark of Porokeratosis is cornoid lamella ${ }^{[4]}$. Cornoid lamella is a column of parakeratotic cells that invaginates the epidermis and corresponds to peripheral keratotic ridge clinically. Beneath this column, stratum granulosum is thinned out or absent. Focus of dyskeratotic cells and vacuolated cells may be present beneath the column. Underlying dermis shows mild lymphoid infiltrate. In our case, biopsy showed only a shallow depression with a small cornoid lamella with dyskeratotic cells beneath it. Porokeratosis is considered as premalignant condition. Though rare, larger, linear and long standing lesions have higher malignant potentiality, especially, in the giant variety ${ }^{[5]}$. Squamous cell carcinoma is the commonest type of malignancy;

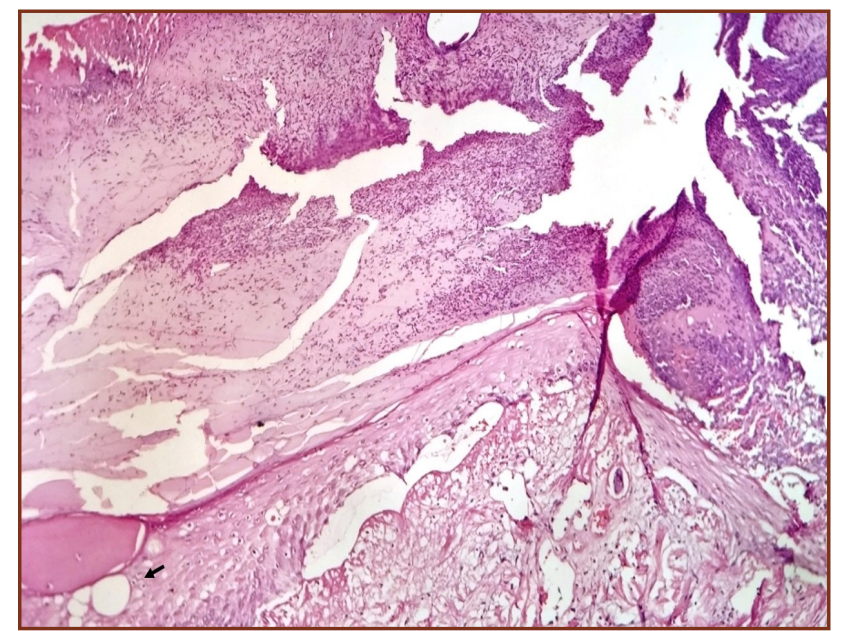

Fig. 2(b): Vacuolation of keratinocytes beneath cornoid lamella (highlighted with an arrow) (10X).

rarely, Bowen's disease and basal cell carcinoma may develop. Porokeratosis in association with lichen planus, diabetes mellitus, CAP syndrome (craniosynostosis, anal anomalies, and porokeratosis), Bloom's syndrome, cystic fibrosis etc have been reported.In our case, patient was having Porokeratosis over scalp which is unusual site to occur and was having squamous cell carcinoma of base of tongue. This could be just mere coincidence or patient might having mucosal lesion of porokeratosis that had undergone malignant transformation. Though the lesion does not match with giant variety of porokeratosis which has high malignant potential, still we have kept the patient under regular follow up for early detection of malignant changes.

No specific treatment modalities have been mentioned for porokeratosis. Many drugs including topical 5-fluorouracil, calcipotriol, 5\% imiquimod and tretinoin have been found effective in the treatment of porokeratosis. Systemic therapy includes use of oral retinoids in patients who are immunosuppressed and are at higher risk for malignant degeneration. Surgical treatment like cryotherapy, dermabrasion, electrodessication, shave and curettage and laser therapy is essential for porokeratosis lesions that have undergone malignant transformation. Protection from sun light/UV radiation and periodic examination of patient for early detection of the malignant changes is mandatory.

\section{Conclusion}

Porokeratosis over scalp is extremely rare condition to occur with and therefore high degree of suspicion and histopathological examination are required for correct diagnosis and management. 


\section{Reference}

1. Pizzichetta MA, Canzonieri V, Massone C, Soyer HP. Clinical and Dermoscopic Features of Porokeratosis of Mibelli. Arch Dermatol 2009;145:91-2.

2. Elisabeth CI, Schreiner W. Porokeratosis. In: Fitzpatrick's Dermatology in General Medicine. 5th ed. New York: McGraw Hill Publication; 1999. p. 624-8.

3. Chen TJ, Chow YC, Chen CH, Kuo TT, Hong HS. Genital porokeratosis: A series of 10 patients and review of the literature. Br J Dermatol 2006;155:325-9.

4. Mukhopadhyay AK. Simultaneous occurrence of disseminated superficial, linear and hypertrophic verrucous forms of porokeratosis in a child. Indian J Dermatol Venereol Leprol 2004;70:364-6.

5. Ghosh SK, Bandyopadhyay D, Chatterjee G, Ghosh AP. Colocalization of lichen planus and porokeratosis of Mibelli. Indian J Dermatol Venereol Leprol 2009;75:532-4.

*Corresponding author:

Dr Rita Vora, Skin Opd 111, Shree krishna Hospital, Karamsad, Anand, Gujarat-388325 (India)

Phone: +91 9879290417

Email: ritavv@charutarhealth.org

Financial or other Competing Interests: None. 